\title{
Fast Estimation of Timing Yield Bounds for Process Variations
}

\author{
Ruiming Chen and Hai Zhou, Senior Member, IEEE
}

\begin{abstract}
With aggressive scaling down of feature sizes in VLSI fabrication, process variation has become a critical issue in designs. We show that two necessary conditions for the "Max" operation are actually not satisfied in the moment matching based statistical timing analysis approaches. We propose two correlationaware block-based statistical timing analysis approaches that keep these necessary conditions, and show that our approaches always achieve the lower bound and the upper bound on the timing yield. Our approach combining with moment-matching based statistical static timing analysis (SSTA) approaches can efficiently estimate the maximal possible errors of moment-matching-based SSTA approaches.
\end{abstract}

Index Terms-Process variations, statistical static timing analysis (SSTA), statistical timing.

\section{INTRODUCTION}

$\mathbf{W}$ ITH aggressive scaling down of feature sizes in VLSI fabrication, process variation has become a critical issue in designs. The corner-based deterministic static timing analysis (STA) becomes pessimistic and inefficient because of the complicated correlations among component delays and the huge number of corners.

The emerging statistical static timing analysis (SSTA) approaches [1]-[7] greatly speed up the analysis by propagating the distributions instead of single values. An essential problem in SSTA is how to compute the maximum of random variables. Assuming that process variations are not very prominent, [3] and [4] used Clark's approach [8] to approximate the maximum of two random variables with Gaussian distribution as a Gaussian variable, and achieved good efficiency and accuracy. Random variables are represented in a linear canonical form, and the first two moments (that is, the mean and the variance) are matched for the maximum. The essential of the approach is the least-squares fitting.

The delay of a gate or a wire is affected by more than one type of process variations, and a linear form may not be accurate enough to capture important information. So, [5]-[7] extended the linear model to nonlinear models. For example, random variables in [7] are represented in a quadratic model. These approaches are shown to be more accurate than those based on the linear model.

Manuscript received January 8, 2007; revised May 1, 2007. This work was supported in part by the National Science Foundation under Grant CCR-0238484 and a Grant from Intel. An earlier version of this paper was presented at the ASP-DAC'07, Yokohama, Japan, January 23-26, 2007.

The authors are with the Department of Electrical Engineering and Computer Science, Northwestern University, Evanston, IL 60208 USA (e-mail: haizhou@eecs.northwestern.edu).

Digital Object Identifier 10.1109/TVLSI.2007.915398

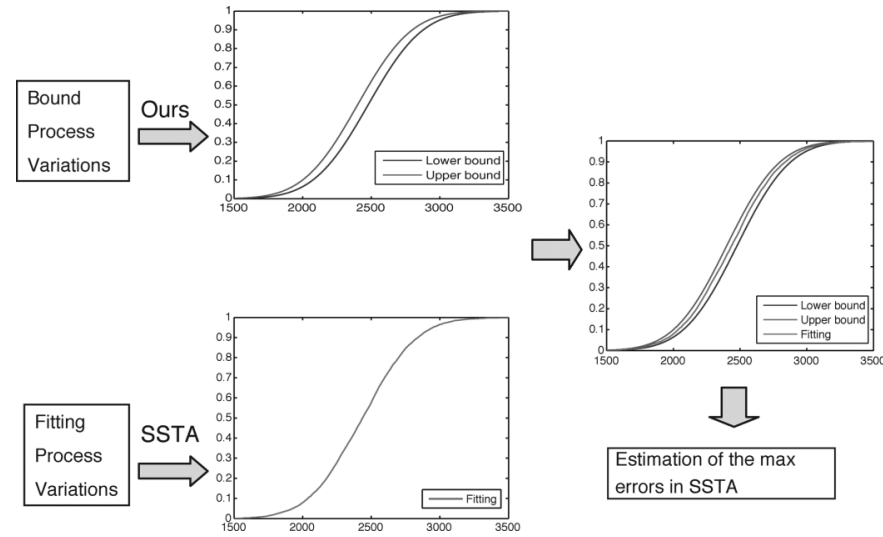

Fig. 1. Our approach is useful for the fast estimation of the maximal errors in moment-matching-based SSTA approaches.

With the development of SSTA tools, many statistical timing optimization works also emerged. These works optimize the timing yield (the probability that a circuit satisfies timing constraints) using SSTA approaches to compute timing information. But how accurate is the timing yield estimation? Without this information, the designers have to over-design in order to make sure that the yield objective is satisfied. Monte Carlo simulation, an expensive approach, is widely used in the existing literatures to verify the results from SSTA. As shown in Fig. 1, if we can efficiently estimate the lower bound and upper bound of the yield, comparing these bounds with the yield estimation by moment matching based SSTA approaches will tell how accurate the estimation is. Agarwal et al. [9], [10] proposed techniques to compute the bounds on yield, but they did not consider correlations: [9] ignored the correlations between components, while [10] ignored the correlations due to path reconvergence, therefore, it is not clear if the computed bounds are close to the actual yield when correlations are considered.

In this paper, we consider how to compute the lower bound and upper bound of timing yield. The existing SSTA works use the linear model or the second order model to approximate process variations. Even the yield computed by the Monte Carlo simulation is not the exact yield. However, the designers can select parameters in the models such that the described process variations are the lower bound or the upper bound of actual process variations. As shown in Fig. 1, our approach computes the bounds of timing yields based on a model that bounds process variations instead of the fitting techniques widely used in literatures. Thus, the accurate computation of the lower and upper bounds of the yield can tell whether the yield objective is satisfied. Enforcing two necessary conditions for the statistical "max" operation that were 
not satisfied by moment-matching-based approaches [3], [4], our approaches achieve tight bounds of yield. Furthermore, for upper bound computation, our techniques can also be used with the second-order model.

The rest of this paper is organized as follows. Section II briefly reviews the existing works on SSTA. Section III presents the relations between the results and the operands in the statistical "Max" operation, and discusses problems in moment-matching-based approaches. Section IV presents our correlation-aware approaches for the statistical "Max" operation. The experiments on the proposed approaches and their comparison with the Monte Carlo simulation are reported in Section V. Finally, the conclusions are drawn in Section VI.

\section{PRELIMINARY}

The combinational circuit is represented by a directed acyclic graph (DAG) $G(V, E)$ with a vertex (or node) set $V$ and an edge set $E$. Each vertex represents a primary input (PI), a primary output (PO), or a gate; each edge represents an interconnection from the source vertex to the target vertex; and the edge weight gives its delay. Two dummy nodes $s$ and $t$ are introduced into the graph: $s$ is connected to all the primary inputs, and $t$ is connected from all the primary outputs. The weights of the edges from $s$ to PIs are the arrival time of the corresponding PIs, and the weights of the edges from POs to $t$ are the negative of the required arrival time at the corresponding POs.

All the delays (or weights), slacks and arrival time are represented in a first-order canonical form as in [3]

$$
c_{0}+\sum_{i=1}^{n} c_{i} X_{i}
$$

where $c_{0}$ is the mean value, $X_{i}$ 's are the principal components [11], and $c_{i}$ 's are their coefficients. Principal component analysis [11] may be performed to get this canonical form [3].

We define the following for two Gaussian random variables $X$ and $Y$ with correlation coefficient $\rho$ :

$$
\begin{aligned}
\phi(x) & =\frac{1}{\sqrt{2 \pi}} \exp \left(-x^{2} / 2\right) \\
\Phi(y) & =\int_{-\infty}^{y} \phi(x) d x \\
\theta_{X Y} & =\sqrt{\sigma_{X}^{2}+\sigma_{Y}^{2}-2 \rho \sigma_{X} \sigma_{Y}} \\
\alpha_{X Y} & =\frac{\mu_{X}-\mu_{Y}}{\theta} .
\end{aligned}
$$

Given any two random variables $X$ and $Y$, [4] defined the tightness probability $T_{X}$ of the variable $X$ as the probability that it is larger than $Y$, and $T_{Y}=1-T_{X}$. Thus

$$
T_{X}=\Phi\left(\frac{x_{0}-y_{0}}{\theta_{X Y}}\right)
$$

when $X \neq Y$.

In block-based SSTA, the moment matching is performed to compute the canonical form representing $\max (X, Y)$. For ex- ample, [4] matches the mean, variance and covariance, while [6] matches the raw moments.

Chang et al. [3] compute the maximal of two Gaussian random variables as follows. Suppose

$$
\begin{aligned}
& A=a_{0}+\sum_{i=1}^{n} a_{i} X_{i} \\
& B=b_{0}+\sum_{i=1}^{n} b_{i} X_{i} .
\end{aligned}
$$

Let $C$ represent $\max (A, B)$. Then according to [8],

$$
\begin{aligned}
\mu(C)= & T_{A} \mu(A)+\left(1-T_{A}\right) \mu(B)+\theta \phi(\alpha) \\
\sigma^{2}(C)= & {\left[\sigma^{2}(A)+\mu^{2}(A)\right] T_{A}+\left[\sigma^{2}(B)+\mu^{2}(B)\right]\left(1-T_{A}\right) } \\
& +[\mu(A)+\mu(B)] \phi(\alpha)-\mu^{2}(C) .
\end{aligned}
$$

The moment matching [3] gives

$$
C=\mu(C)+\frac{\sigma(C)}{s_{0}} \sum_{i} \beta_{i} X_{i}
$$

where

$$
\begin{aligned}
\beta_{i} & =T_{A} a_{i}+\left(1-T_{A}\right) b_{i} \\
s_{0} & =\sqrt{\sum_{i} \beta_{i}^{2}} .
\end{aligned}
$$

\section{STATISTICAL "MAX” OPERATION}

We introduce two concepts for relations among random variables.

Definition 1 (Dominance Relation): A random variable $A$ dominates variable $B$ when

$$
\operatorname{Pr}(A \geq B)=1
$$

Definition 2 (Comparison Relation): A random variable $C$ has comparison relation with variables $A$ and $B$ when

$$
\begin{aligned}
& \operatorname{Pr}(C>A)=\operatorname{Pr}(B>A) \\
& \operatorname{Pr}(C>B)=\operatorname{Pr}(A>B) .
\end{aligned}
$$

The following theorem shows that both the dominance relation and the comparison relation are necessary conditions for the statistical "Max" with its operands.

Theorem 1: Suppose $A$ and $B$ are random variables and $C=$ $\max (A, B)$, then $C$ dominates $A$ and $B$, and has the comparison relations with them.

Proof: Since $C$ is the maximum of $A$ and $B, C \geq A$, and $C \geq B$, so $C$ dominates $A$ and $B$

$$
\begin{aligned}
\operatorname{Pr}(C>A) & =\operatorname{Pr}(\max (A, B)-A>0) \\
& =\operatorname{Pr}(\max (A-A, B-A)>0) \\
& =\operatorname{Pr}(\max (0, B-A)>0) \\
& =\operatorname{Pr}(B-A>0) .
\end{aligned}
$$


Similarly, we can prove

$$
\operatorname{Pr}(C>B)=\operatorname{Pr}(A>B)
$$

The block-based SSTA approaches [3], [4] assume that all the random variables have Gaussian distribution. They use a canonical form

$$
c_{0}+\sum_{i=1}^{n} c_{i} X_{i}
$$

to represent a random variable, where $c_{0}$ is the nominal value and $X_{i}$ 's are independent random variables with standard normal distribution. When they compute the maximum of Gaussian variables, they use Clark's approach [8] to match the mean and the variance. But during this match, the dominance and comparison relations are not kept. For example, compute the maximum of the following two Gaussian random variables using the approach in [4]:

$$
\begin{aligned}
& A=30+x_{1} \\
& B=30.5+0.5 x_{1} .
\end{aligned}
$$

Suppose $C=\max (A, B)$, then theoretically,

$$
\operatorname{Pr}(C \geq A)=1 \quad \operatorname{Pr}(C \geq B)=1 .
$$

But the results computed from the moment matching based approach in [4] are

$$
\operatorname{Pr}(C \geq A)=89.46 \% \quad \operatorname{Pr}(C \geq B)=62.57 \% .
$$

So the dominance relations are not kept. Also, theoretically

$$
\operatorname{Pr}(A>B)=15.84 \%
$$

but the moment-matching-based approach gets

$$
\operatorname{Pr}(C>B)=62.57 \%
$$

which has a big difference from $\operatorname{Pr}(A>B)=15.84 \%$. So the comparison relations are not kept either.

We also took the approach in [6] to approximate the maximum of two Gaussian variables as a non-Gaussian variable, and find that neither the dominance nor comparison relation is kept. For example, using the approach in [6], for the dominance relations, we get

$$
\operatorname{Pr}(C \geq A)=63.43 \% \quad \operatorname{Pr}(C \geq B)=49.17 \%
$$

and for the comparison relations, we get

$$
\operatorname{Pr}(C>B)=49.17 \% \neq \operatorname{Pr}(A>B)=15.84 \% .
$$

Thus, the existing approximation approaches have not kept the necessary conditions in the statistical "Max" operation.

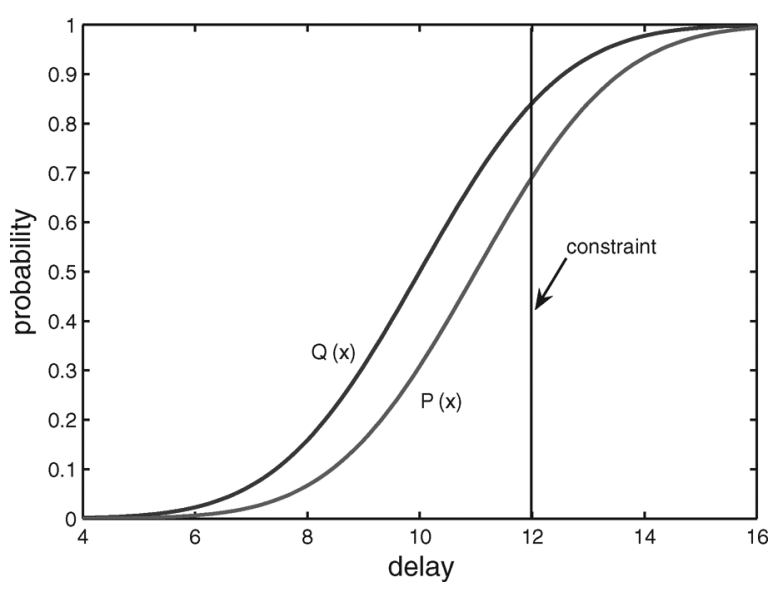

Fig. 2. $\operatorname{CDF} Q(x)$ is an upper bound of $\operatorname{CDF} P(x)$.

A timing analysis approach may be used in timing optimizations. In statistical timing optimization, we need to compute the yield, that is, the probability that the constraint is satisfied. Since the moment-matching-based SSTA approaches [3], [4] are approximation approaches, there is no guarantee whether they are conservative or optimistic. For example, given a timing constraint for the maximal delay from the primary input to the primary output, we do not know if the computed yield is larger or smaller than the actual yield.

Definition 3: For any two cumulative distribution functions $P(x)$ and $Q(x), Q(x)$ is the upper bound of $P(x)$ (and $P(x)$ is the lower bound of $Q(x)$ ) if and only if $\forall x: Q(x) \geq P(x)$. As shown in Fig. 2, using the upper bound of $P(x)$, the yield $\operatorname{Pr}(x \leq$ constraint $)$ according to the upper bound of $P(x)$ is higher than the yield according to $P(x)$. We will show later that the approaches based on the dominance relations or the comparison relations give the lower bound or the upper bound of the yield, respectively.

\section{SSTA Without Moment Matching}

"Max" and "Add" are the two fundamental operations in timing analysis. In SSTA, all random variables are represented in the canonical form. The "Add" operation is simple and exact. For the "Max" operation, we plan to enforce either the dominance relation or the comparison relation.

\section{A. Theory}

Our SSTA approach traverses a circuit in the topological order and computes the distribution of the arrival time at each node. Depending on what relations the procedure keeps, our approach has two variants. The first one, denoted as LBDomSSTA, keeps the dominance relations, while the second one, denoted as UBCompSSTA, keeps the comparison relations.

Note that the theory in this subsection holds for random variables of any distributions, not only limited to Gaussian.

For the dominance relation, we have the following theorem. 
Theorem 2: In a combinational circuit, when a "max" operation is encountered, if a random variable that dominates the operands is used for their maximum, the computed yield will be a lower bound of the actual yield.

Proof: Suppose $A$ and $B$ are operands of the "max" operation, and $C$ dominates $A$ and $B$. Then $C \geq A$ and $C \geq B$, so $C \geq \max (A, B)$. If $C$ is used as the maximum of $A$ and $B$, the computed maximal delay is no less than the actual maximal delay, so the yield is not larger than the actual yield.

Therefore, LBDomSSTA is guaranteed to get the lower bound of yield.

The comparison relations can be transformed into

$$
\begin{aligned}
& \operatorname{Pr}(C \leq A)=\operatorname{Pr}(B \leq A) \\
& \operatorname{Pr}(C \leq B)=\operatorname{Pr}(A \leq B) .
\end{aligned}
$$

For the comparison relation, we have the following.

Theorem 3: Suppose $A$ and $B$ are two random variables, and let

$$
C=\beta A+(1-\beta) B
$$

where $\beta \in[0,1]$, then $C$ always satisfies the comparison conditions

$$
\begin{aligned}
& \operatorname{Pr}(C \leq A)=\operatorname{Pr}(B \leq A) \\
& \operatorname{Pr}(C \leq B)=\operatorname{Pr}(A \leq B) .
\end{aligned}
$$

Now we prove the following lemma.

Lemma 1: Suppose $A$ and $B$ are two random variables, and

$$
C=\beta A+(1-\beta) B
$$

where $\beta \in[0,1]$, then

$$
\max (A, B) \geq C \text {. }
$$

\section{Proof:}

$$
\begin{aligned}
\max (A, B)-C= & \max (A-C, B-C) \\
= & \max (A-(\beta A+(1-\beta) B), \\
& B-(\beta A+(1-\beta) B)) \\
= & \max ((1-\beta)(A-B), \beta(B-A)) .
\end{aligned}
$$

Thus, if $A \geq B,(1-\beta)(A-B) \geq 0$, so $\max (A, B) \geq C$; if $A \leq B, \beta(B-A) \geq 0$, so $\max (A, B) \geq C$.

According to Lemma 1, we know that the "max" of two random variables as computed in Theorem 3 is not larger than their actual maximum. Therefore, we have the following lemma based on the monotonicity property of the "max" operation.

Lemma 2: The maximal delay from the primary inputs to the primary outputs computed in UBCompSSTA is not greater than the actual maximal delay.
For two random variables $A$ and $B$, if $P_{r}(A \leq B)=1$, then $P_{r}(A \leq D) \geq P_{r}(B \leq D)$ for any constant $D$. Thus, we have the following theorem.

Theorem 4: The yield computed by UBCompSSTA gives the upper bound of the actual yield.

\section{B. Lower Bound}

Most of the existing SSTA approaches assume that random variables have Gaussian distributions. In this subsection, we consider the LBDomSSTA under this assumption. Suppose $A$ and $B$ are two Gaussian variables. Let

$$
\begin{aligned}
& A=a_{0}+\sum_{i=1}^{n} a_{i} x_{i} \\
& B=b_{0}+\sum_{i=1}^{n} b_{i} x_{i} \\
& C=\max (A, B) \approx c_{0}+\sum_{i=1}^{n} c_{i} x_{i} .
\end{aligned}
$$

Unfortunately, we have the following theorem.

Theorem 5: Let $A$ and $C$ be two Gaussian variables represented in the first-order canonical form. Then

$$
\operatorname{Pr}(C \geq A)=1
$$

cannot be satisfied unless $C=A+d$ with $d$ a non-negative constant number.

\section{Proof: Suppose}

$$
\begin{aligned}
& A=a_{0}+\sum_{i=1}^{n} a_{i} X_{i} \\
& C=c_{0}+\sum_{i=1}^{n} c_{i} X_{i} .
\end{aligned}
$$

$$
\text { If } C=A+d
$$

$$
\operatorname{Pr}(C \geq A)=\operatorname{Pr}(A+d \geq A)=\operatorname{Pr}(d \geq 0)=1 .
$$

If $C \neq A+d$, obviously $\sum_{i=1}^{n}\left(a_{i}-c_{i}\right)^{2} \neq 0$. So

$$
\begin{aligned}
\operatorname{Pr}(C \geq A) & =\Phi\left(-\mu_{A-C} / \sigma_{A-C}\right) \\
& =\Phi\left(-\frac{a_{0}-c_{0}}{\sqrt{\sum_{i=1}^{n}\left(a_{i}-c_{i}\right)^{2}}}\right) .
\end{aligned}
$$

But $\Phi(x)$ is not equal to 1 , so

$$
\operatorname{Pr}(C \geq A)<1 .
$$


Therefore, it is almost impossible to find a Gaussian random variable to dominate all the operands simultaneously. However, if the dominance relation is relaxed to

$$
\operatorname{Pr}(C \geq A) \geq \eta \quad \operatorname{Pr}(C \geq B) \geq \eta
$$

with $0<\eta<1$, it is possible to find a $C$ satisfying this condition. With increasing $\eta$, we have increasing confidence that the computed yield is a lower bound.

Clark [8] stated that the covariance between $C=\max (A, B)$ and any random variable $Y$ can be expressed in terms of covariances between $A$ and $Y$ and between $B$ and $Y$ as

$$
\operatorname{Cov}(C, Y)=\operatorname{Cov}(A, Y) T_{A}+\operatorname{Cov}(B, Y)\left(1-T_{A}\right) .
$$

As suggested in [4], in order to preserve the covariance, for every $Y=x_{i}$, we must have

$$
c_{i}=a_{i} T_{A}+b_{i}\left(1-T_{A}\right), \quad i=1,2, \ldots n .
$$

Our approach adjusts the mean value $\left(c_{0}\right)$ to satisfy the dominance relations given by the following inequalities:

$$
\begin{aligned}
& \operatorname{Pr}(C \geq A)=\Phi\left(\frac{c_{0}-a_{0}}{\left(1-T_{A}\right) \sqrt{\sum_{i}\left(a_{i}-b_{i}\right)^{2}}}\right) \geq \eta \\
& \operatorname{Pr}(C \geq B)=\Phi\left(\frac{c_{0}-b_{0}}{T_{A} \sqrt{\sum_{i}\left(a_{i}-b_{i}\right)^{2}}}\right) \geq \eta .
\end{aligned}
$$

Let $\zeta$ be a constant satisfying $\Phi(\zeta)=\eta$. Then the two inequalities can be transformed to

$$
\begin{aligned}
\frac{c_{0}-a_{0}}{\left(1-T_{A}\right) \sqrt{\sum_{i}\left(a_{i}-b_{i}\right)^{2}}} & \geq \zeta \\
\frac{c_{0}-b_{0}}{T_{A} \sqrt{\sum_{i}\left(a_{i}-b_{i}\right)^{2}}} & \geq \zeta .
\end{aligned}
$$

By solving this inequality set, we can find the minimal $c_{0}$. The dominance relations are then satisfied.

\section{Upper Bound}

1) Gaussian Variables: In this subsection, we also assume that random variables have Gaussian distributions. According to Theorem 3 and the discussion in Section IV-B, if we select $\beta=T_{A}$, the comparison relations are kept, and the covariance is also preserved.

In order to check whether the upper bound is tight, we compare the mean and the variance computed by our approach and those by the moment-matching-based approach. Let $C$ represent the maximum of the two Gaussian random variables $A$ and $B$ computed by our approach, and $D$ represent $\max (A, B)$ computed by [8]

$$
\begin{aligned}
\mu(D)-\mu(C)= & \left(T_{A} \mu_{A}+\left(1-T_{A}\right) \mu_{B}+\theta \phi(\alpha)\right) \\
& -\left(T_{A} \mu(A)+\left(1-T_{A}\right) \mu(B)\right) \\
= & \theta \phi(\alpha) \geq 0 .
\end{aligned}
$$

Assuming that all the random variables have at most $10 \%$ deviation $(3 \sigma)$ from their nominal values, we get

$$
\begin{aligned}
\theta^{2}= & \sigma^{2}(A)+\sigma^{2}(B)-2 \rho \sigma(A) \sigma(B) \\
\leq & \sigma^{2}(A)+\sigma^{2}(B)+2 \sigma(A) \sigma(B) \\
\leq & (0.10 \mu(A) / 3)^{2}+(0.10 \mu(B) / 3)^{2} \\
& +2(0.10 \mu(A) / 3)(0.10 \mu(B) / 3) \\
\leq & (0.10 / 3)^{2}(\mu(A)+\mu(B))^{2} .
\end{aligned}
$$

Thus

$$
\begin{aligned}
\frac{\theta^{2}}{\mu^{2}(D)} & \leq \frac{\theta^{2}}{\left(T_{A} \mu_{A}+\left(1-T_{A}\right) \mu_{B}\right)^{2}} \\
& \leq(0.10 / 3)^{2} \frac{(\mu(A)+\mu(B))^{2}}{\left(T_{A} \mu(A)+\left(1-T_{A}\right) \mu(B)\right)^{2}} .
\end{aligned}
$$

Since the random variables in our problem represent delay or arrival time, if we set the arrival time at the PIs to 0 , their mean values should be non-negative. Without loss of generality, we assume $\mu(A) \geq \mu(B)>0$. So $T_{A} \geq 0$.5. Let $\mu(A)=\gamma \mu(B)$, so $\gamma \geq 1$. Thus

$$
\begin{aligned}
\frac{\theta^{2}}{\mu^{2}(D)} & \leq(0.10 / 3)^{2} \frac{(\gamma \mu(B)+\mu(B))^{2}}{\left(\gamma T_{A} \mu(B)+\left(1-T_{A}\right) \mu(B)\right)^{2}} \\
& =(0.10 / 3)^{2} \frac{(1+\gamma)^{2}}{\left(\gamma T_{A}+1-T_{A}\right)^{2}} \\
& \leq(0.10 / 3)^{2} \frac{(1+\gamma)^{2}}{(0.5+0.5 \gamma)^{2}} \\
& =4(0.10 / 3)^{2} \\
& =0.0044 .
\end{aligned}
$$

In addition, $\phi(\alpha) \leq 1 / \sqrt{2 \pi}$, so the relative error of the mean is at most

$$
\frac{\sqrt{0.0044}}{\sqrt{2 \pi}}=2.66 \% \text {. }
$$

From this derivation, we can see that if the variance is smaller, or the correlation is positive and larger, the result will be more accurate. In practice, this relative error is even smaller because of the highly positive correlation between delays and the small variance.

Even though the mean plays a major role in the yield, we will also estimate the error on the variance

$$
\begin{aligned}
\sigma^{2}(D)-\sigma^{2}(C)= & T_{A}\left(1-T_{A}\right)\left[\sigma^{2}(A)+\sigma^{2}(B)-2 \rho \sigma(A) \sigma(B)\right. \\
& \left.+\left(a_{0}-b_{0}\right)^{2}\right]-\theta^{2} \phi^{2}(\alpha) \\
& +\theta \phi(\alpha)\left[\left(a_{0}-b_{0}\right)\left(1-2 T_{A}\right)\right]
\end{aligned}
$$

If $\theta=0$

$$
\begin{aligned}
0 & =\sqrt{\sigma^{2}(A)+\sigma^{2}(B)-2 \rho \sigma(A) \sigma(B)} \\
& =\sqrt{\sum_{i}\left(a_{i}-b_{i}\right)^{2}} .
\end{aligned}
$$


Thus

$$
\begin{aligned}
a_{i} & =b_{i} \quad \forall i=1, \ldots, n \\
T_{A} & =0 \text { or } T_{A}=1 .
\end{aligned}
$$

So

$$
\sigma^{2}(D)-\sigma^{2}(C)=0
$$

If $\theta>0$ (note $\theta \geq 0$ )

$$
\begin{aligned}
\alpha^{2} & =\frac{(\mu(A)-\mu(B))^{2}}{\sigma^{2}(A)+\sigma^{2}(B)-2 \rho \sigma_{A} \sigma_{B}} \\
& \geq \frac{(\gamma-1)^{2}}{(0.10 / 3)^{2}(\gamma+1)^{2}} \\
& =900 \frac{(\gamma-1)^{2}}{(\gamma+1)^{2}} .
\end{aligned}
$$

According to [12], when $\alpha \geq 3$, the right-hand side of (16) approaches 0 . So when $\gamma \geq 1.22$, the error approaches 0 .

When $\gamma<1.22$, according to [12]

$$
\sigma^{2}(D)-\sigma^{2}(C) \leq 0.091 \theta^{2}
$$

While according to (15), when $\rho \geq 0$ and $\gamma<1.22$

$$
\theta^{2} \leq 0.0022 \mu^{2}(D)
$$

Thus

$$
\begin{aligned}
\sigma^{2}(D)-\sigma^{2}(C) & \leq 0.091 * 0.0022 \mu^{2}(D) \\
& =0.0002 \mu^{2}(D) .
\end{aligned}
$$

Therefore

$$
\frac{\sigma^{2}(D)-\sigma^{2}(C)}{\mu^{2}(D)} \leq 0.02 \%
$$

Therefore, the error on variance is at most $0.02 \%$ of the square of mean value. If the correlation coefficient $(\rho)$ is more positive, this error gets even smaller. For example, when $\rho=1$, the error is less than $0.01 \%$. Note that moment-matching-based approaches are doing approximations, so the errors computed here are not the errors from the actual values.

In summary, the results computed in UBCompSSTA are very close to the results from the moment-matching approach. Note that the moment-matching approach is also an approximation approach, so it is possible for UBCompSSTA to have better results than the moment-matching approach.

2) Non-Gaussian Variables: In this part, we consider the cases where the random variables do not have Gaussian distributions. The delay of a gate or an interconnect may be affected by not only one kind of process variation, so there may exist nonlinear relations between the delays and the process variations. For example, the delay of a wire is affected by the process variations on the wire length, the wire width, and the wire thickness. Zhang et al. [7] has proposed a quadratic delay model for a wire. The delay random variable $D$ is represented in the following quadratic model:

$$
D=m+\alpha \delta+\delta^{T} \Upsilon \delta+\gamma^{T} r
$$

where $r=\left(R_{1}, R_{2}, \ldots, R_{p}\right)^{T}$ represents the local variances, $\delta=\left(X_{1}, X_{2}, \ldots, X_{q}\right)^{T}$ represents the global variances, $\alpha$ and $\gamma$ are sensitivity vectors, and $\Upsilon$ is a sensitivity matrix. All these $R_{i}$ 's and $X_{j}$ 's are independent and have the standard Gaussian distribution. The random variables represented in this form do not have a Gaussian distribution. We will show that our approach also gets results close to the results from [7].

Suppose random variables $A$ and $B$ are represented in the quadratic model

$$
\begin{aligned}
& A=m_{A}+\alpha_{A} \delta+\delta^{T} \Upsilon_{A} \delta+\gamma_{A}^{T} r \\
& B=m_{B}+\alpha_{B} \delta+\delta^{T} \Upsilon_{B} \delta+\gamma_{B}^{T} r .
\end{aligned}
$$

In the computation of the maximum of two random variables $A$ and $B$ represented in the quadratic model, Zhang et al. [7] approximated the random variables as Gaussian variables by moment matching and computed the skewness of the output. If the skewness is greater than a threshold, the "max" operation is delayed, otherwise, the approach got

$$
\max (A, B)=m_{C}+\alpha_{C} \delta+\delta^{T} \Upsilon_{C} \delta+\gamma_{C}^{T} r
$$

where

$$
\begin{aligned}
m_{C} & =T_{A} m_{A}+\left(1-T_{A}\right) m_{B}+\theta \phi(\alpha) \\
\alpha_{C} & =T_{A} m_{A}+\left(1-T_{A}\right) m_{B} \\
\Upsilon_{C} & =T_{A} \Upsilon_{A}+\left(1-T_{A}\right) \Upsilon_{A} \\
\gamma_{C} & =T_{A} \gamma_{A}+\left(1-T_{A}\right) \gamma_{B} .
\end{aligned}
$$

The output of our approach differs from the output of [7] only in the $m$ part. Our approach gets

$$
m=T_{A} m_{A}+\left(1-T_{A}\right) m_{B} .
$$

Since $m$ affects only the mean value, we only need to compute the difference on the mean value in our approach. Let $C$ and $D$ represent the outputs of [7] and our approach, respectively. The difference between $\mu(C)$ and $\mu(D)$ is $\theta \phi(\alpha)$. We can show similarly that the relative error of the mean is at most $2.66 \%$.

Thus, our approach can also be applied to methods where the variables do not have Gaussian distributions, and get an upper bound of the yield that is close to the result from [7].

\section{EXPERIMENTAL RESULTS}

We have implemented LBDomSSTA and UBCompSSTA in $\mathrm{C}++$. Experiments were performed on the large cases in ISCAS85 benchmark. We use the cases where all the random variables have the Gaussian distributions as examples to show the effectiveness of our approaches. We also implemented the Monte Carlo simulation to compute the maximal delay from $s$ to $t$. We made 10000 trials for each test case. All the random 
TABLE I

Comparison Results of UBCompSSTA, LBDomSSTA, [3] AND Monte CARLo Simulation on LinEar Model

\begin{tabular}{|c|c|c|c|c|c|c|c|c|c|c|c|c|c|c|}
\hline \multirow[t]{2}{*}{ name } & \multicolumn{4}{|c|}{ UBCompSSTA } & \multicolumn{4}{|c|}{ LBDomSSTA } & \multicolumn{3}{|c|}{ Monte Carlo } & \multicolumn{3}{|c|}{$[3]$} \\
\hline & time $(\mathrm{s})$ & $\mu$ & $\sigma$ & yield $(\%)$ & time $(\mathrm{s})$ & $\mu$ & $\sigma$ & yield $(\%)$ & $\mu$ & $\sigma$ & yield $(\%)$ & $\mu$ & $\sigma$ & yield $(\%)$ \\
\hline $\mathrm{c} 1355$ & 0.01 & 1580 & 40 & 91.15 & 0.01 & 1585 & 40 & 89.07 & 1583 & 40 & 90.00 & 1583 & 40 & 89.62 \\
\hline c1908 & 0.01 & 4000 & 100 & 91.92 & 0.01 & 4019 & 101 & 88.49 & 4011 & 100 & 90.00 & 4018 & 101 & 88.49 \\
\hline c2670 & 0.01 & 2918 & 61 & 91.15 & 0.01 & 2926 & 61 & 89.25 & 2922 & 61 & 90.00 & 2923 & 61 & 89.80 \\
\hline c3540 & 0.03 & 4700 & 120 & 92.22 & 0.02 & 4727 & 120 & 88.30 & 4715 & 119 & 90.00 & 4718 & 120 & 89.44 \\
\hline c5315 & 0.03 & 4900 & 123 & 91.47 & 0.02 & 4919 & 123 & 88.69 & 4910 & 125 & 90.00 & 4913 & 123 & 89.25 \\
\hline c6288 & 0.03 & 12400 & 312 & 92.36 & 0.03 & 12477 & 314 & 87.90 & 12443 & 313 & 90.00 & 12464 & 314 & 88.69 \\
\hline c7552 & 0.05 & 4300 & 107 & 91.47 & 0.04 & 4320 & 107 & 88.30 & 4311 & 107 & 90.00 & 4313 & 107 & 89.44 \\
\hline
\end{tabular}

variables have at most $10 \%$ deviation from their nominal values. All the experiments were run on a Linux PC with a $2.4 \mathrm{GHz}$ Xeon CPU and 2.0 GB memory.

The comparison results of UBCompSSTA, LBDomSSTA, [3], and the Monte Carlo simulation are shown in Table I. We perform Monte Carlo simulations to compute the $90 \%$ percentile point of the maximal delay from $s$ to $t$, and select this point as the timing constraint. We have $\eta=90 \%$ in LBDomSSTA. The columns $2-5$ show the running time, the mean of the maximal delay, the standard deviation of the maximal delay, and the yield computed by UBCompSSTA, respectively. The columns 6-9 show the running time, the mean of the maximal delay, the standard deviation of the maximal delay, and the yield computed by LBDomSSTA, respectively. The 10th and 11th columns show the mean and the standard deviation of the maximal delay from the Monte Carlo simulation, respectively. The results show that our approaches always get tight bounds of the yield. The errors on the yield are $1.68 \%$ and $1.43 \%$ on average for UBCompSSTA and LBDomSSTA, respectively. The relative errors on the mean and the variance are also quite small. The [3] gets more accurate results than ours since it is doing the fitting instead of the bounding. The significant contribution of our work is that it provides an efficient way to estimate the maximal errors of the SSTA approaches without doing the expensive Monte Carlo simulation. For example, for these testcases, if we do not know the Monte Carlo simulation results, we can also conclude that [3] gets very accurate results since the yields from [3] are very close to the bounds computed from our approaches.

Fig. 3 shows the cumulative distribution functions from LBDomSSTA, UBCompSSTA, and the Monte Carlo simulation for the case "c6288". The CDF from UBCompSSTA stays on the left side, while the CDF from LBDomSSTA stays on the right, and the actual CDF stays between them. It demonstrates that our approaches achieve the bounds in the whole range.

UBCompSSTA is also tested based on the quadratic model. Table II shows the comparison results between UBCompSSTA and the Monte Carlo simulation. The results show that UBCompSSTA has an error of $1.50 \%$ on average.

\section{CONCLUSION}

The state-of-the-art statistical static timing analysis approaches cannot tell whether the computed yield is smaller or larger than the actual yield. In this paper, we proposed two block-based statistical static timing analysis approaches by satisfying each of the two necessary conditions for "max"

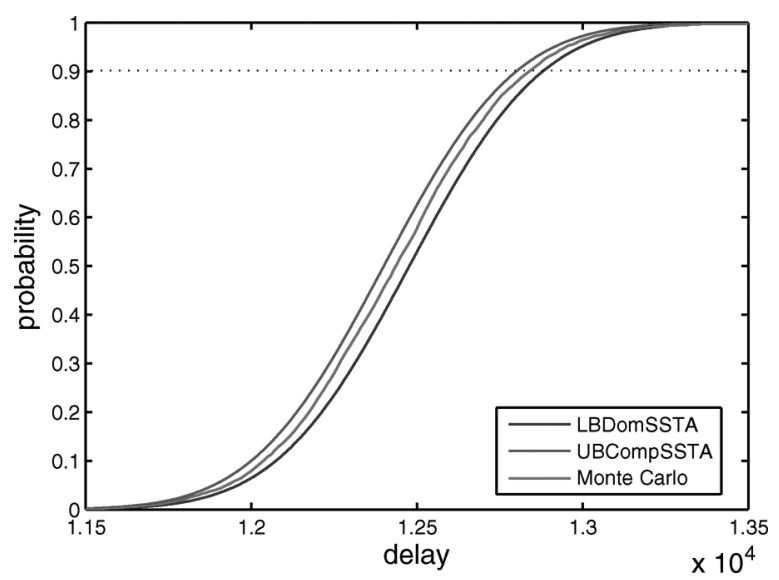

Fig. 3. CDFs from different approaches for "c6288".

TABLE II

COMPARISON RESUlTS OF UBCompSSTA AND MONTE CARLO SIMULATION ON QUADRATIC MODEL

\begin{tabular}{||r||r|r|r||r|r|r|r||}
\hline \multicolumn{1}{||c||}{ name } & \multicolumn{3}{|c||}{ Monte Carlo } & \multicolumn{4}{|c||}{ UBCompSSTA } \\
\cline { 2 - 8 } & $\mu$ & $\sigma$ & yield (\%) & time $(\mathrm{s})$ & $\mu$ & $\sigma$ & yield (\%) \\
\hline c1355 & 1629 & 75 & 90.00 & 1.18 & 1623 & 73 & 90.97 \\
\hline c2670 & 4088 & 151 & 90.00 & 1.54 & 4073 & 142 & 91.37 \\
\hline c2670 & 2960 & 78 & 90.00 & 0.63 & 2949 & 76 & 92.39 \\
\hline c3540 & 4810 & 169 & 90.00 & 3.05 & 4786 & 168 & 91.63 \\
\hline c5315 & 4997 & 168 & 90.00 & 6.14 & 4979 & 165 & 91.48 \\
\hline c6288 & 12700 & 472 & 90.00 & 5.14 & 12623 & 442 & 92.16 \\
\hline c7552 & 4397 & 156 & 90.00 & 5.99 & 4378 & 152 & 90.52 \\
\hline
\end{tabular}

operation. We showed that our approach achieves a tight upper-bound of the actual yield. Furthermore, for the upper bound computation, our approach achieves the bound even without the assumption of Gaussian distributions for the random variables.

\section{REFERENCES}

[1] M. Orshansky and K. Keutzer, "A general probabilistic framework for worst case timing analysis," in Proc. Des. Autom. Conf., 2002, pp. 556-561.

[2] J. A. G. Jess, K. Kalafala, S. R. Naidu, R. Otten, and C. Visweswariah, "Statistical timing for parametric yield prediction of digital integrated circuits," in Proc. Des. Autom. Conf., 2003, pp. 932-937.

[3] H. Chang and S. S. Sapatnekar, "Statistical timing analysis considering spatial correlations using a single pert-like traversal," in Proc. Int. Conf. Comput.-Aided Des., 2003, pp. 621-625.

[4] C. Visweswariah, K. Ravindran, K. Kalafala, S. G. Walker, and S. Narayan, "First-order incremental block-based statistical timing analysis," in Proc. Des. Autom. Conf., 2004, pp. 331-336.

[5] H. Chang, V. Zolotov, S. Narayan, and C. Visweswariah, "Parameterized block-based statistical timing analysis with non-gaussian parameters, nonlinear delay functions," in Proc. Des. Autom. Conf., 2005, pp. $71-76$. 
[6] Y. Zhan, A. J. Strojwas, X. Li, L. T. Pileggi, and D. Newmark, "Correlation-aware statistical timing analysis with non-gaussian delay distributions," in Proc. Des. Autom. Conf., 2005, pp. 77-82.

[7] L. Zhang, W. Chen, Y. Hu, J. A. Gubner, and C. C. Chen, "Correlation-preserved non-Gaussian statistical timing analysis with quadratic timing model," in Proc. Des. Autom. Conf., 2005, pp. 83-88.

[8] C. E. Clark, "The greatest of a finite set of random variables," Oper. Res., vol. 9, no. 2, pp. 145-162, 1961.

[9] A. Agarwal, D. Blaauw, V. Zolotov, and S. Vrudhula, "Computation and refinement of statistical bounds on circuit delay," in Proc. Des. Autom. Conf., 2003, pp. 348-349.

[10] A. Agarwal, D. Blaauw, and V. Zolotov, "Statistical timing analysis for intra-die process variations with spatial correlations," in Proc. Int. Conf. Comput.-Aided Des., 2003, pp. 900-907.

[11] W. J. Krzanowski, Principles of Multivariate Analysis. London, U.K.: Oxford Univ. Press, 2000.

[12] D. Sinha, N. Shenoy, and H. Zhou, "Statistical gate sizing for timing yield optimization," in Proc. Int. Conf. Comput.-Aided Des., 2005, pp. $1037-1041$

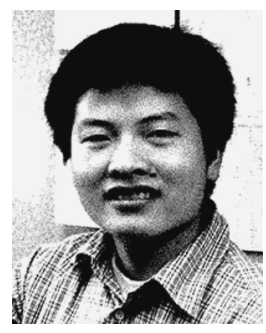

Ruiming Chen received the B.S. degree in microelectronics and the M.S. degree in computer science from Tsinghua University, Beijing, China, in 2000 and 2003, respectively. He is currently pursuing the $\mathrm{Ph} . \mathrm{D}$. degree in computer engineering from Northwestern University, Evanston, IL.

His research interests include VLSI computer-aided design (CAD), especially on buffer insertion and statistical timing.

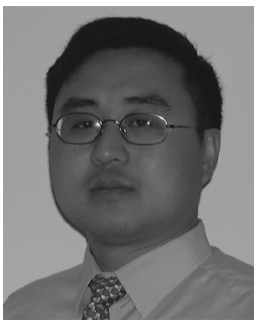

Hai Zhou (SM'04) received the Ph.D. degree in computer sciences from the University of Texas at Austin, in 1999, and the B.S. and M.S. degrees in computer science and technology from Tsinghua University, Beijing, China, in 1992 and 1994, respectively.

He is an Associate Professor with the Department of Electrical Engineering and Computer Science, Northwestern University, Evanston, IL. His research interests include VLSI computer-aided design, algorithm design, and formal methods. He has published more than 70 technical papers in prestigious journals and conferences in these areas.

Prof. Zhou was a recipient of the CAREER Award from the National Science Foundation in 2003. He served on the technical program committees of ACM Design Automation Conference, IEEE International Conference on ComputerAided Design, and many other conferences. 